\title{
Improving Family Medicine in Kosovo with Microsystems
}

\author{
Donald O. Kollisch, MD, Cristina S. Hammond, MPH, Ellen Thompson, MS, RN, \\ and James Strickler, $M D$
}

Purpose: Family medicine is being adopted in many low-income countries to meet medical care needs. A systems approach may be useful for international organizations offering aid, in addition to providing resources and training. An established methodology called Microsystems was used to help implement family medicine in Kosovo, a small country seeking to rebuild after decades of turmoil and war.

Methods: Clinical and systems changes were implemented in 2 municipalities, resulting in improved quality of care within the established primary care system. The first 2-year project focused on hypertension and the second on antenatal care. Mutual exchanges were used to introduce Microsystems, addressing medical records, data systems, evidence-based guidelines, community outreach, supplemental training, and sustainability models.

Results: The Microsystems method successfully guided specific clinical, general management, and organizational improvements. Successes included improved teamwork; delivery of patient-centered care; empowered nursing staff; and data-driven decision making. Barriers to systems change included management systems impeding staff initiative; resistance to change by the larger health care "macrosystem"; marginal funding for prevention; and few models for clinical prevention and continuity care.

Conclusions: Microsystems methods are adaptable for use in low-income countries or those rebuilding after conflict that are implementing family medicine models to improve medical care and population health. (J Am Board Fam Med 2011;24:102-111.)

Keywords: Delivery of Health Care, Family Medicine, International Health, Kosovo, Microsystems

Many low-income countries are seeking to improve health care by following a family medicine model, ${ }^{1-3}$ embracing a team approach that is patient centered and data driven. ${ }^{4}$ Family physicians from wealthier

This article was externally peer reviewed.

Submitted 17 May 2010; revised 8 October 2010; accepted 18 October 2010.

From the Department of Community Health and Social Medicine, Sophie Davis School of Biomedical Education, The City College of New York, New York (DOK); the Department of Community and Family Medicine (CSH, JS), Dartmouth Medical School, Hanover, NH; and the Dartmouth-Hitchcock Medical Center, Lebanon, NH (ET).

Funding: This work was supported by 2 grants from the United States Agency for International Development. 2004-2006 Dartmouth Medical School-Gjakova, Kosovo AIHA Reproductive Health Partnership (USAID) \#UEEKOS04PPTRHAGA00, 2002-2004 Dartmouth Medical School-Gjilan, Kosovo AIHA Primary Care Partnership (USAID) \#UEEKOS-02-PPTRHAGJ-00. Both partnership projects were administered through the American International Health Alliance, a not-for-profit organization.

Conflict of interest: none declared.

Corresponding author: Donold O. Kollisch, MD, Donald. O.Kollisch@Dartmouth.edu systems, such as those in Western Europe and the United States, are increasingly providing support to countries with fewer resources. ${ }^{5}$ Most such aid programs focus primarily on training to improve providers' knowledge and skills and on delivering additional material resources, such as equipment and medications. ${ }^{6}$

More than that is needed, however. ${ }^{7}$ It is becoming more recognized that attention to the systems of care is needed to effectively meld training and resources $^{8}$ (Figure 1). The use of clinical quality improvement methods, such as Microsystems, can facilitate implementation by focusing on the development of efficient office structures, data use, and management styles. There is already evidence that Microsystems can be adapted for use outside of well-established settings in the West. ${ }^{9}$

\section{Context and Historical Background}

Kosovo was a province of southern Serbia within the former Yugoslavia until declaring indepen- 
Figure 1. Microsystems approach to implementing and sustaining healthcare change. Reproduced with permission from Little GA, Hammond CS. International perspectives: the Kosovo-Dartmouth Alliance for Healthy Newborns. NeoReviews 2008;9:e233-41. Copyright 2008 by the AAP.

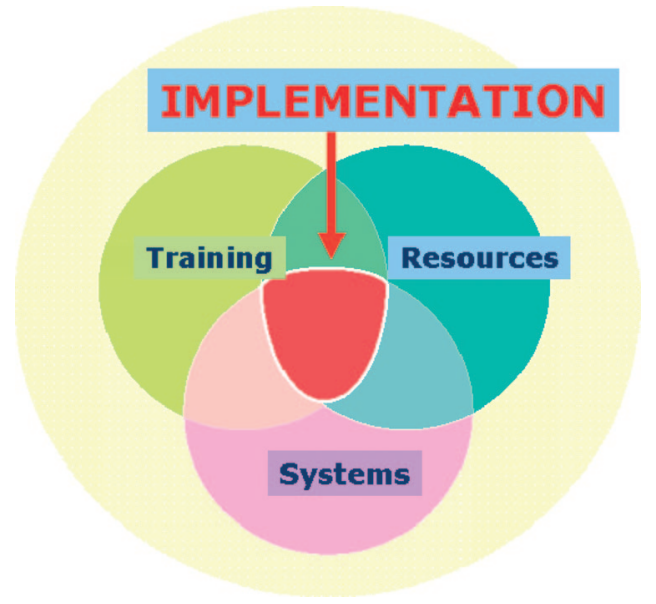

continued to see patients in the "old" way, with patients massing in front of their office doors, seeking referrals to specialists for acute problems; nurses functioned primarily as administrative personnel. There were few systems for preventive services or for management of chronic disease. $\mathrm{Pa}$ tients had limited expectations of this new family medicine.

Following the goals of the newly functioning $\mathrm{MoH}$, a team from the Dartmouth Medical School in the United States partnered with the FMCs to improve primary care implementation. Two projects linked health professionals from Dartmouth with family medicine counterparts in one of Kosovo's 30 municipalities. The Gjilan municipality, home to approximately 120,000 people, served from 2001 to 2003 as the pilot of Microsystems methodologies. Based on this successful partnership, a second project was developed from 2004 to 2006 in the Gjakova municipality, with a population of approximately 140,000 .

dence in February 2008. Health care for its approximately 2 million people was disordered after a decade of ethnic violence, a war, and an 8-year period of United Nations administration. ${ }^{10}$ The staff of Kosovo's family medicine centers (FMCs) recognized that their health care system-like those developing in other nations-faced many challenges, including poorly functioning management; pervasive poverty; minimal professional resources; lack of well-trained staff; underfunding of the health sector; and a population with a pressing need for comprehensive health care. A program of reform was developed by the new Ministry of Health $(\mathrm{MoH})$ with major assistance and guidance from United Nations agencies, the World Health Organization (WHO) and the European Agency for Reconstruction. ${ }^{11,12}$ This plan included a family medicine model to deliver primary care. To prepare, a series of family medicine training programs were developed for doctors and nurses. ${ }^{13,14}$

\section{The Problem}

Despite training in modern family medicine, professional staff found it difficult to implement fullservice care, largely because systems of care delivery continued to resemble the old Yugoslavian systems. ${ }^{13,15}$ There existed a top-down management hierarchy, few medical records, and little continuity of care. Newly minted family physicians

\section{The Model}

Microsystems

Clinical Microsystems ${ }^{16,17}$ is a practical approach to organizing the staff of a health care delivery system, such as an ambulatory clinic or hospital, to analyze their work from multiple perspectives and make plans to incorporate improvements. Work processes are looked at primarily from the patient's viewpoint to measure patient-centered outcomes. The perspectives of staff, including satisfaction, training, and resources, are also measured and improvements considered. Although each clinic or hospital is unique, the Microsystems approach has identified some common elements, including patient flow, communication among staff, patient education, resource allocation, and evidence-based care. ${ }^{18,19}$ Effective use of Microsystems depends on working in teams, developing leadership, using simple and understandable measures of outcomes, and involving the entire staff in the process.

We applied Microsystems to our partnerships in Kosovo by having a few training seminars followed by working with the leadership to convene groups of doctors and nurses to address each of the discrete work processes (Figure 2). Each group launched "Plan-Do-Check-Act" initiative cycles to seek incremental improvements, and all efforts were coordinated by a joint nurse/doctor leadership group. 
Figure 2. Improving primary health care using a Microsystems approach.

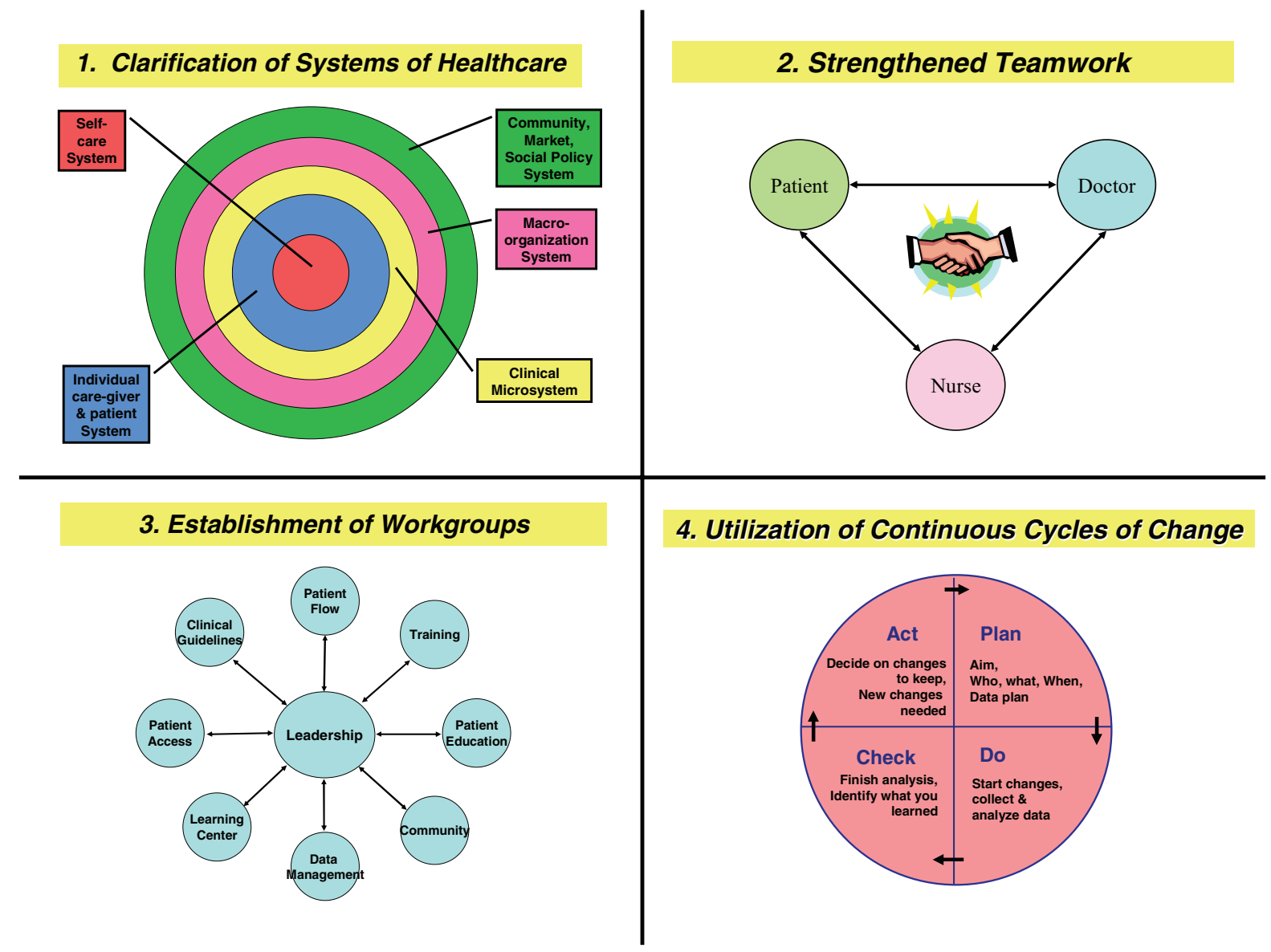

Peer exchanges between Kosovo and the United States focused on building leadership and teamwork capabilities, sharing quality improvement methodology, assessing needs, and identifying barriers. Kosovar partners' experiences in US FMCs allowed them to identify "best practices" that could be adapted for Kosovo and helped solidify their vision of their own health care system.

It was determined by the Committee for the Protection of Human Subjects (at Dartmouth) and by the $\mathrm{MoH}$ (in Kosovo) that this quality improvement project was exempt from institutional review board review.

\section{Gjilan: Chronic Disease Management}

The Gjilan leadership group decided to target one common chronic health condition. Hypertension was chosen because it would foster doctor-nurse teamwork and promote prevention. It also had the benefit of being clinically well defined and easy to measure. The equipment needed to detect and monitor hypertension (stethoscopes and sphygmomanometers) was relatively inexpensive, and medications to treat hypertension (hydrochlorothiazide, atenolol, captopril) were already on Kosovo's "Essential Medication" list. Finally, the doctors and nurses of Gjilan believed that chronic disease was an increasingly important indicator of health in their developing nation, in addition to maternalchild health and infectious disease. ${ }^{20}$

Outcome goals and indicators were established for disease management and community health promotion:

- Increase professional staff knowledge and skills.

- Identify resources needed to screen for, diagnose, and treat hypertension.

- Improve care infrastructure, including patient flow, professional role clarification, record-keeping, data collection systems, and team management.

- Increase the proportion of adult patients screened. 
The leadership group developed training modules for doctors and nurses to enhance basic clinical skills. Blood pressure was previously measured by a doctor only if a patient had "symptoms" of hypertension, ie, chest pain, headache, or palpitations. Now, every adult would have their blood pressure measured by a nurse during every visit. The workgroups focused on specific tasks. One formulated clinical practice guidelines for both chronic hypertension and hypertensive emergencies. Another created medical records products, including "triage" cards to document 3 elevated readings; continuity forms to record core medical history and physical findings; and flow sheets to monitor hypertension management. Other groups worked on developing and implementing screening programs in community settings, such as schools, "pensioner" centers, and rural FMCs; reviewing formulary guidelines; and establishing data collection, analysis, and reporting systems.

\section{Gjakova: Antenatal Care in Community-Based Clinics}

The project in family medicine in Gjakova was directed to use Microsystems to improve reproductive health. Antenatal care was chosen by the Gjakova leadership group for 2 major reasons. The first is that the infant mortality rate of 55 out of every 1000 after the 1999 war was thought to be the highest in Europe. It had come down to approximately 35 out of every 1000 at the initiation of the project in 2004, still approximately 5-fold higher than in the nations of Western Europe. The birth rates and maternal-child morbidity of the Gjakova region were thought to be representative of Kosovo as a whole. ${ }^{21}$

The second was that many women-especially those from rural areas-received little or no antenatal care, often limited to a single ultrasound to determine sex and to be sure that the baby was "developing properly." Antenatal care was delivered by obstetricians, primarily in the larger cities. There were no traditional birth attendants and few community-based midwives. Most women delivered in a hospital with an obstetrician attending, but they arrived there with little preparation. Although the MoH had declared FMCs to be the location for free antenatal care for all women, this simply was not taking place. There were no standards, clinical guidelines, practical implementation plans, or resources to help with the delivery of antenatal care.
Fortunately, the WHO had recently described an effective 4-visit model of antenatal care. ${ }^{22,23}$ This model was inexpensive, low-tech, and could be readily adapted for a decentralized, communitybased family medicine system.

Using this model, the Gjakova leadership group developed a vision for antenatal care that could be implemented within the limits of the family medicine Microsystem.

Outcome goals and indicators were established for antenatal care, including:

- Increase the knowledge and skills of the Family medicine staff to provide high quality antenatal care.

- Develop and secure the resources needed to provide antenatal care in the FMCs.

- Establish Microsystems-based quality improvement to support sustainability.

- Develop strategic alliances with local obstetricians.

As in Gjilan, the Microsystems approach was used to help the Gjakova leadership develop the processes needed for antenatal care. The leadership group was initially coached by their counterparts from Gjilan, which permitted them to avoid some inefficiencies and dead ends. Workgroups were established with aims and objectives that were more refined than those used in Gjilan: patient flow, patient education, training, data, community, and specialist alliance. A timeline was developed for training the staff and distributing low-cost resources (eg, fetoscopes, urine dipsticks, posters, patient education materials). Guidelines, training resources, and patient education materials were developed, often adapting work that had been previously published and tested. ${ }^{24-27}$ Patient, family, community, and staff surveys were conducted to help understand the baseline reality of the FMCs and the existing patterns of antenatal care. Fortuitously, there were several strong obstetric advocates in Gjakova who supported antenatal care by family physicians.

Though the Dartmouth team spent fewer weeks in Gjakova than they had in Gjilan with the previous project, and therefore had less time to monitor and guide the workgroups, it became clear that strong local commitment to improving the health of mothers and babies through implementing antenatal care resulted in continued progress between 
visits. In addition, Dartmouth worked closely with the FMC medical director on leadership skills; the medical director's persistence and guidance were vital.

\section{Effects of Change}

Both of the Dartmouth-Kosovo partnerships successfully used Microsystems methods to guide improvements. Though each used a single clinical condition-one hypertension and the other pregnancy—as a vehicle for change, the projects had a broad impact on patient focus, teamwork, data use, outcomes measurement, and efficient office system redesign throughout the municipality's FMCs.

\section{Gjilan}

The partnership was successful in achieving many of the indicators for specific workgroups, as outlined in Table 1. Ninety-four percent of doctors (61 of 65 ), and $97 \%$ of nurses (152 of 156) employed by the FMCs completed clinical training and passed Objective Structured Clinical Examinations (OSCE) to establish competency. Medical records were developed, approved, printed, and adopted. All adult patients presenting for primary care at all FMCs in Gjilan were screened for hypertension and received nurse-led patient education. At the main FMC, small but significant improvements in blood pressure were documented for 140 hypertensive patients who presented for care between November 2002 and September 2003. The 53 patients who returned for at least one follow-up visit showed significant decreases in both systolic and diastolic blood pressure measurements from the first visit to the last recorded visit (mean systolic change, $12 \mathrm{~mm}$ $\mathrm{Hg}$; 95\% CI, 6-17; mean diastolic change, $5 \mathrm{~mm}$ Hg; 95\% CI, 1-9).

Community-based blood pressure screening clinics-never before done in Kosovo-began in February 2003 and continue to date. In the first 10 community screenings, 860 people participated and $24 \%$ were found by nurses to have hypertension in the WHO categories of moderate, severe, or very severe. They all were verified by a physician, received patient counseling from nurses, and were referred to their FMC for management.

A notable success was the strengthened role of nurses. As nursing skills were upgraded, nurses took on more responsibilities in areas such as patient assessment, patient education, and supervi- sion. The increased morale, professionalism, and support, as well as improved patient flow, were noted by patients and resulted in increased satisfaction for nurses and doctors, as measured using before-and-after surveys. ${ }^{28}$

Throughout the project, partners worked to develop strong relationships with local, regional, and nationwide leaders and organizations. The $\mathrm{MoH}$ adopted the partnership's Hypertension Clinical Practice Guideline for use throughout Kosovo, ${ }^{29}$ and a "Dissemination Conference," conducted at the conclusion of the partnership in the capital city of Prishtina, was attended by family physicians from 23 of the 30 municipalities.

Despite changing leadership, a labile political "macrosystem," and shifting priorities, the main FMC continues to follow the screening and treatment protocols, to use the patient flow and medical records systems, and to function with doctor-nurse teams. In addition, Microsystem working group methods used for hypertension were extended to diabetes care, including community outreach, patient education, and treatment protocols.

\section{Gjakova}

Because FMCs had not previously provided antenatal care in Kosovo, there was little confidence that women would come to the FMCs for care. The initial target set by the leadership group was to enroll a minimum of 25 pregnant women. Enrollment began in 2 FMCs, one rural and one urban, and then was rolled out in the remaining 7 FMCs over 5 months in 2005. One of the inaugural FMCs, led by a doctor-nurse team who were energetically engaged and committed, enrolled $79 \mathrm{pa}-$ tients. Over 10 months a total of 172 women enrolled for antenatal care, representing at least 7\% of the pregnant women in Gjakova in that period. Antenatal charts, antenatal "passports," and multiple patient education brochures were developed and used at all 9 centers. Low-cost resources, including fetal stethoscopes, Dopplers, measuring tapes, proteinuria test strips, and gestational wheels, were procured and distributed by the Gjakova leadership.

Audits performed at all 9 FMCs demonstrated provision of antenatal care in accordance with the WHO standards. ${ }^{22,23}$ Achievement of standards for indicators such as blood pressure measurement, weight measurement, delivery of key patient education messages, and appropriate referral to obstet- 


\begin{tabular}{|c|c|c|}
\hline $\begin{array}{l}\text { Improvement } \\
\text { Workgroup }\end{array}$ & Aim & Outcome \\
\hline Training & $\begin{array}{l}\text { Continuously increase the knowledge } \\
\text { and skills of health providers at the } \\
\text { MFMC }\end{array}$ & $\begin{array}{l}\text { - } 94 \% \text { ( } 61 \text { of } 65) \text { doctors and } 97 \% \text { (152 of } 156) \text { nurses trained } \\
\text { and demonstrating competency for blood pressure measurement } \\
\text { and HTN management, including patient education } \\
\text { - Ongoing monthly CPD programs led by and for staff doctors } \\
\text { and nurses; clinical topics selected by group consensus }\end{array}$ \\
\hline Patient flow & $\begin{array}{l}\text { To have most functional flow of } \\
\text { patients through the MFMC }\end{array}$ & $\begin{array}{l}\text { - Redesigned use of space with clearly designated areas for } \\
\text { registration, patient waiting, screening, clinical care, and } \\
\text { medical records } \\
\text { - Increased patient satisfaction from March through October } \\
2003\end{array}$ \\
\hline $\mathrm{CPG}$ & $\begin{array}{l}\text { Develop, adapt, and use guidelines } \\
\text { for doctors and nurse to identify } \\
\text { and manage HTN in patients at } \\
\text { the MFMC }\end{array}$ & $\begin{array}{l}\text { - CPG for HTN created by and for team of doctors and } \\
\text { nurses (baseline, no HTN CPG) } \\
\text { - HTN CPG used at MFMC } \\
\text { - } 144 \text { hypertensive patients identified and followed with new } \\
\text { protocol including medical record } \\
\text { - HTN CPG adopted by MoH for use throughout Kosovo }\end{array}$ \\
\hline Data & $\begin{array}{l}\text { Design and use data forms and } \\
\text { databases in order to improve the } \\
\text { care of patients with HTN }\end{array}$ & $\begin{array}{l}\text { - Forms created and in use: medical record for HTN; patient } \\
\text { HTN "passport"; daily patient registration datasheets, patient } \\
\text { and staff surveys (baseline, } 0 \text { medical records or passports) } \\
\text { - Registration data collected for more than } 40,000 \text { patient } \\
\text { visits during year } 1 \text { (baseline, } 0 \text { ) } \\
\text { - Data shared and used to inform and improve care via data } \\
\text { display in patient areas and routine reporting to leadership } \\
\text { group }\end{array}$ \\
\hline Patient education & $\begin{array}{l}\text { Provide the best health education } \\
\text { (about HTN) for the population } \\
\text { of Gjilan }\end{array}$ & $\begin{array}{l}\text { - Nurses provide patient education for all patients found at } \\
\text { screening to have high blood pressure or be at risk for HTN } \\
\text { (baseline, no nurse-led patient education) } \\
\text { - Patient education brochures created and distributed at } \\
\text { individual appointments and in waiting area (baseline, no } \\
\text { available materials) } \\
\text { - Patient education videotapes available for various health } \\
\text { topics }\end{array}$ \\
\hline LRC & $\begin{array}{l}\text { Increase knowledge and skills of the } \\
\text { MFMC staff through better use of } \\
\text { information systems (computer, } \\
\text { Internet, library resources) }\end{array}$ & $\begin{array}{l}\text { - } 5 \text { computers with Internet connection available at MFMC for } \\
\text { medically related use by staff and interested community } \\
\text { members (baseline, no LRC) } \\
\text { - Doctors and nurses trained by LRC staff: } 117 \text { in computer } \\
\text { use, } 56 \text { in basic Internet searching, } 15 \text { in evidence-based } \\
\text { medical searching } \\
\text { - } 1 \text { Clinical Practice Standard Review completed on } \\
\text { hypertensive crisis (baseline, 0) }\end{array}$ \\
\hline Patient access & $\begin{array}{l}\text { Improve quality of care by having } \\
\text { patients get their care at the } \\
\text { MFMC in their own community }\end{array}$ & $\begin{array}{l}\text { - Increase in Monday staffing in response to higher volume } \\
\text { - } 24 \% \text { decrease ( } 31 \% \text { in November } 2002 \text { to } 7 \% \text { in October } \\
2003 \text { ) of patients seen at MFMC rather than family medicine } \\
\text { center in their own community } \\
\text { - } 52 \% \text { decrease in visits resulting in antibiotic injection } \\
\text { (average of } 268 \text { per week in November } 2002 \text { to } 139 \text { per week } \\
\text { in March 2003) }\end{array}$ \\
\hline Community outreach & $\begin{array}{l}\text { Use hypertension screening as a } \\
\text { vehicle to build a good } \\
\text { relationship between the MFMC } \\
\text { and the community }\end{array}$ & $\begin{array}{l}\text { - } 10 \text { community screenings for HTN held in } 8 \text { months; } \\
\text { provided screening for } 860 \text { individuals; counseling and } \\
\text { referral for appropriately identified patients } \\
\text { - Ongoing patient education radio and TV spots for various } \\
\text { common health topics, including HTN } \\
\text { - Patient education posters for HTN in community settings }\end{array}$ \\
\hline
\end{tabular}

AIHA, American International Health Alliance; MFMC, main family medicine center; CPD, Continuing Professional Development; CPG, clinical practice guideline; HTN, hypertension; LRC, learning resource center (a tool promoted and supported by AIHA partnerships); MoH, Minstry of Health.

ric specialists ranged from $83 \%$ to $95 \%$ (Figure 3). Chart reviews conducted on all antenatal care charts indicated excellent compliance with quality protocols.
There also was strong staff and patient satisfaction with the care delivered, which was measured with before-and-after surveys. Patient interviews indicated appreciation for the education and the 
Figure 3. Gjakova family medicine centers antenatal care clinical and educational service indicators.

\section{Gjakova Family Medicine Centers Antenatal Care Clinical and Educational Service Indicators}

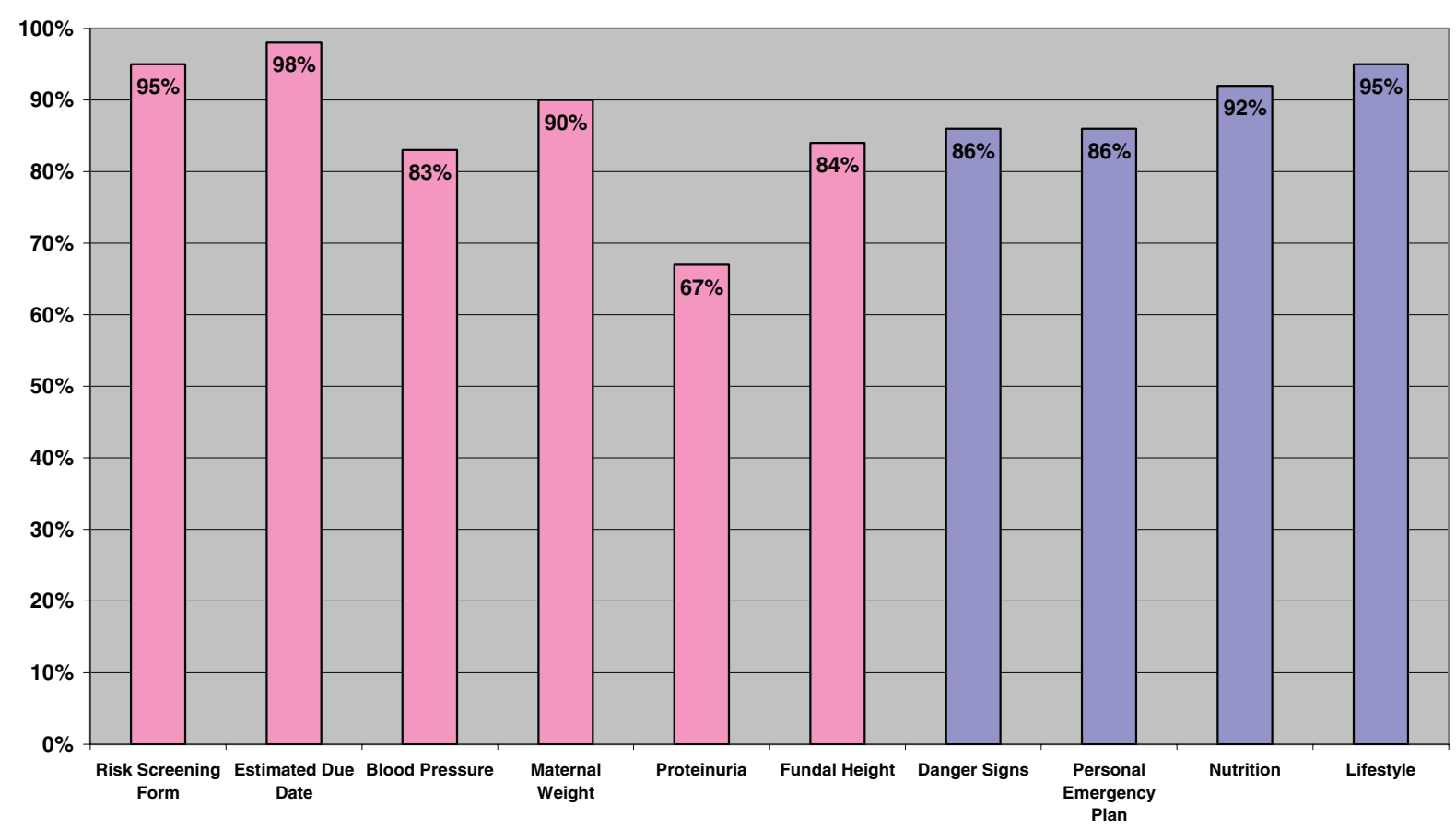

time spent with them. Staff were pleased to be providing important care and indicated a strong desire to sustain this initiative over time. Alliances were forged with key local obstetricians; continued effort was needed to sustain these relationships and assure good communication at community and hospital levels.

\section{Discussion}

The principles of quality improvement and $\mathrm{Mi}-$ crosystems lend themselves well to use in international settings. Implementing new models of care in a country that is underdeveloped, resource-challenged, or traumatized requires building capacity from the inside if it is to be accepted and sustained. The use of internal leadership can create positive staff focus; optimize roles for efficient care; foster trust, teamwork, and self-worth; and empower all staff to accomplish and improve their work. This approach stresses community outreach and involvement as well as alliances with the political and cultural organizations whose support and engagement are crucial for guidance and sustainability. ${ }^{5}$
To promote sustainable health care changes for a country in transition, consultants should strengthen local capacity for performing needs assessments and implementing, evaluating, and sustaining change. In Kosovo, family physicians and nurses needed to integrate systems for highquality services into their routine work for these services to be delivered consistently and measurably. Because primary care is inclusive rather than disease specific, it has the capacity to flexibly respond to community health needs.

The key change Kosovo faced was to make a transition from the Soviet style of dogmatic, topdown management to one more nimble and responsive to staff initiative and patients' needs. The Microsystem model attempted to institute a patient-centered care system in which there was previously a system driven by the needs of the staff. Family medicine had been accepted at the policy level, and Microsystems represented a strategy for implementation of that model.

In these projects, the US physicians and nurses offered their expertise in the development of rational and sustainable systems for change, but they did 
- 162 family medicine doctors and nurses completed antenatal care training

- 9 family medicine centers implemented antenatal care in Gjakova municipality (100\%); staggered roll-out over 10 months

- 172 women received 254 antenatal care visits at family medicine centers ( $7 \%$ of population)

- $25 \%$ early, appropriate identification of risk and referral to specialist (consistent with WHO-model expectations)

- $28 \%$ of eligible women returned for postpartum visits

- Antenatal care included clinical and educational care (see Figure 3)

- Patient education highly valued by both patients and staff

- Staff found provision of care "very rewarding"

- Staff formed good relationships with patients

- Staff developing relationships with obstetric colleagues

WHO, World Health Organization.

not provide direct clinical service or substantial new resources. Education was limited to "training the trainers," and all of the local physicians and nurses were trained by Kosovars.

In both regions, the Dartmouth consultants initially acted to spark and fuel changes to improve care. As each project evolved, their role shifted to that of advisor and coach. Sustainability depended on reaching a threshold for change such that the local doctors and nurses would not turn back. In Gjilan this has resulted in blood pressure screening now being the standard of care. In Gjakova, the family medicine system now includes antenatal care as a "standard" service, although underfunding limits the acquisition of even low-cost supplies.

Based on the Gjakova project, a USAID-funded Global Development Alliance disseminated the successful antenatal care pilot project to 27 FMCs in 9 municipalities and also introduced improvements in neonatal intensive care. ${ }^{30}$

The lessons that we learned as consultants in Kosovo may have broad applicability for systems support in other resource-challenged settings
(Table 3). These experiences and conclusions are consistent with a recent comprehensive report on Kosovo by Physicians for Human Rights. ${ }^{31}$

\section{Conclusion}

Improving primary health care in post-war Kosovo presented challenges that may be encountered in regions with political instability, damaged infrastructure, and limited resources. The Microsystems approach-focusing on team-building, systems of care, and data-based decision making-proved effective in Kosovo to address both maternal health and chronic illness care. Strengthening primary care settings $^{32}$ with Microsystem improvements will likely result in successes in leadership, teamwork, and satisfaction, thus enabling primary care to be the focal point for improving health care in other low-resource, developing, or post-conflict countries.

We thank the staff and leadership of the FMCs in Gilan and Gjakova for their enthusiastic work. In particular, Dr. Hajriz Ibrahimi, Dr. Jashar Ramadani, and Nurse (now Dr.) Ramize Ibrahimi of Gjilan; and Dr. Basri Komoni, Dr. Zef Komani, and

\section{Table 3. Kosovo-Dartmouth Partnership: Lessons Learned}

- Change in culture from specialty care to primary care will take time and attention from the Ministry of Health.

- Time lags for funding impact project momentum, supply chain, and reinforcement of new practice patterns.

- Lack of system-wide health statistics on which to base our outcome data makes true outcome data very difficult to collect and rely on.

- Care processes that do not include systematic follow-up or preventive care reflect ongoing cultural change challenges that will take time and attention from providers and the healthcare system of the country.

- Changes in leadership within the family medicine centers led to interruption of progress in improvements made and threatened to move the family medicine centers back to previous standards.

- Lack of funding for the healthcare system overall, including for supplies and professional salaries, has had a tremendous impact on the project. Professionals are unable to live at current salary levels and must have second/third jobs to survive. This had led to many requests for additional payment for work done within the project and increased tension. It has also led to a lack of basic supplies of equipment and medications necessary to care for the population. 
Nurse Keni Koshi of Gjakova were essential to the success of the partnerships. We gratefully acknowledge the support of the Ministry of Health and of Dr. Genc Ymerhalili of the Center for Development of Family Medicine in Kosovo; Dr. Mary Taylor, international quality improvement expert and consultant to our project; and the many doctors and nurses affiliated with Dartmouth for their volunteer assistance.

\section{References}

1. Gunn JM, Palmer VJ, Naccarella L, et al. The promise and pitfalls of generalism in achieving the AlmaAta vision of health for all. Med J Aust 2008;189: 110-2.

2. Christianson CE, Bistrovsky VF, Kogut BM. Family medicine in the Russian Far East. Fam Med 2007; 39:742-5.

3. Abyad A, Al-Baho AK, Unluoglu I, et al. Development of family medicine in the Middle East. Fam Med 2007;39:736-41.

4. van Weel C, Rosser WW. Improving health care globally: a critical review of the necessity of family medicine research and recommendations to build research capacity. Ann Fam Med 2004;2(Suppl2): S5-16.

5. Abraham S. Country-centered family medicine. Fam Med 2008;40:234-5.

6. American Academy of Family Medicine. International family medicine. Available at: http://www.aafp. org/online/en/home/aboutus/specialty/international. html. Accessed September 24, 2010.

7. Murray SA. Out of Africa: some lessons for general practice/family medicine in developed countries? Fam Prac 2000;17:361-3.

8. Boelen C, Haq C, Hunt V, Rivo M, Shahady E. Improving health systems: the contribution of family medicine. A guidebook. Singapore: World Organization of Family Doctors; 2002.

9. Little GA, Hammond CS. International perspectives: the Kosovo-Dartmouth Alliance for Healthy Newborns. NeoReviews 2008;9:e233-41.

10. Jones SG, Hilborne LH, Anthony CR, et al. Securing health: lessons from nation-building missions. Santa Monica, CA: Rand Corporation; 2006.

11. Sejdiu P, Vuori H. Health policy for Kosovo. Prishtina, Kosovo : Department of Health and Social Welfare, Joint Interim Administration Structure, United Nations Mission in Kosovo; 2001.

12. Figueras J, McKee M, Cain J, Lessof S, eds. Health systems in transition (2004). World Health Organization, European Observatory on Health Systems and Policies. 2004. Available at: http://www.euro. who.int/en/what-we-publish/abstracts/health-systemsin-transition-learning-from-experience. Accessed September 24, 2010.

13. Hedley R, Maxhuni B. Development of family medicine in Kosovo. BMJ 2005;331:201-3.

14. Morikawa MJ. Primary care training in Kosovo. Fam Med 2003;35:440-4.
15. Bobak M, Pikhart H, Rose R, Hertzman C, Marmot M. Socioeconomic factors, material inequalities, and perceived control in self-rated health: cross-sectional data from seven post-communist countries. Soc Sci Med 2000;51:1343-50.

16. Nelson EC, Batalden PH, Huber TP, et al. Microsystems in health care: Part 1. Learning from highperforming front-line clinical units. Jt Comm J Qual Improv 2002;28:472-93.

17. Clinical Microsystems [Homepage]. Available at: http://www.clinicalmicrosystems.org. Accessed September 24, 2010.

18. Nelson E, Batalden P, Godfrey M. Quality by design: a clinical microsystems approach. San Francisco, CA: Jossey-Bass; 2007.

19. Quinn JB. Microsystem management as a promising new methodology for improving the cost and quality of health care. Jt Comm J Qual Improv 2002;28:495-6.

20. Yach O, Hakes C, Gould CL, Hofman KJ. The global burden of chronic diseases: overcoming impediments to prevention and control. JAMA 2006; 291:2616-22.

21. Lulaj S, Gashi A, Çela L, et al. Perinatal situation in Kosovo for years 2000-2004. Available at: http:// www.unicef.org/kosovo/kosovo_media_pub_survival. 003.04.pdf. Accessed September 24, 2010.

22. Caroli G, Villar J, Piaggio G, et al. WHO systematic review of randomised controlled trials of routine antenatal care. Lancet 2001;357:1565-70.

23. Villar J, Ba'aqeel H, Piaggio G, et al. WHO antenatal care randomised trial for the evaluation of a new model of routine antenatal care. Lancet 2001; 357:1551-64.

24. Kinzie B, Gomez P, Chase R. Basic maternal and newborn care: a guide for skilled providers. Available at: http://www.jhpiego.org/resources/pubs/mnh/ BMNCrevmanEN.pdf. Accessed September 24, 2010.

25. World Health Organization. Making pregnancy safer. Pregnancy, childbirth, postpartum and newborn care: a guide for essential practice. Available at: http://www.who.int/making_pregnancy_safer/ documents/924159084x/en/index.html. Accessed September 24, 2010.

26. The White Ribbon Alliance for Safe Motherhood/India. Saving mothers' lives: what works. Field guide for implementing best practices in safe motherhood. Available at: http://www.whiteribbonalliance.org/Resources/ Documents/Complete\%20PDF\%20Saving\%20Mothers \%20Lives\%20-\%20What\%20Works.pdf. Accessed September 24, 2010.

27. March of Dimes Foundation Web site. Pregnancy and Newborn Health Education Center also Resources for Professionals. Available at: http://www. marchofdimes.com. Accessed September 24, 2010.

28. Thompson E, Harding AL, Pond F, Hammond C, Taylor M. A partnership to improve health care in Kosovo: Dartmouth Medical School and Kosovar nurses worked together on a two-year project to re- 
build primary health care in the post conflict city of Gjilan. Am J Nurs. 2006;106:72CC-DD,72FF,72HH.

29. Ramadani J, Carkaxhiu L, Kastrati A, et al. [Standard guidelines for the treatment of hypertension]. Prishtina, Kosovo: Center for the Development of Family Medicine, Ministry of Health; 2004.

30. Homan FF, Hammond CS, Thompson EF, Kollisch DO, Strickler JC. Post-conflict transition and sustainability in Kosovo: establishing primary health- care based antenatal care. Prehosp Disaster Med 2009;25:18-23.

31. Physicians for Human Rights. Perilous medicine: the legacy of conflict and oppression on health in Kosovo. June 2009. Available at: http://physiciansforhumanrights.org/ library/documents/reports/perilous-medicine.pdf. Accessed September 24, 2010.

32. Starfield B. Global health, equity, and primary care. J Am Board Fam Med 2007;20:511-3. 